\title{
Topological Indices of Some Classes of Thorn Complete and Wheel Graphs
}

\author{
Divyashree B K ${ }^{1}$, Jagadeesh $\mathbf{R}^{2, *}$, Siddabasappa ${ }^{3}$ \\ 1 Department of Mathematics, Government Science College, Bangalore University, Bengaluru 560001, India; \\ divyashree1704@gmail.com (D.B.K.); \\ 2 Department of Mathematics, Government First Grade College, Ramanagar-562159, Karnataka, India; \\ jagadeeshr1978@gmail.com (J.R.); \\ 3 Department of Mathematics, Government Science College, Bangalore University, Bengaluru 560001, India; \\ siddabasappa1961@gmail.com (S.); \\ * Correspondence: jagadeeshr1978@gmail.com;
}

Received: 20.01.2021; Revised: 1.05.2021; Accepted: 5.05.2021; Published: 27.06.2021

\begin{abstract}
We have multiple real numbers that describe chemical descriptors in the field of Graph theory. These descriptors constitute the entire structure of a graph, which possesses an actual chemical structure. Among these, the main focus of topological indices is that they are associated with many nonidentical physiochemical properties of chemical compounds. Also, the biological properties of chemical compounds can be established by the topological indices. In this analysis, we compute the Reciprocal Randic index $\left(R^{-1}\right)$, Reduced Reciprocal Randic index $\left(R R^{-1}\right)$, Atom-bond Connectivity index $(A B C)$ and the geometric arithmetic index $(G A)$ of thorn graphs are obtained theoretically.
\end{abstract}

Keywords: Reciprocal Randic $\left(R^{-1}\right)$ index; Reduced Reciprocal Randic $\left(R R^{-1}\right)$ index; Atom-bond Connectivity $(A B C)$ index; Geometric-arithmetic $(G A)$ index; cog-graph; thorn graph.

(C) 2021 by the authors. This article is an open-access article distributed under the terms and conditions of the Creative Commons Attribution (CC BY) license (https://creativecommons.org/licenses/by/4.0/).

\section{Introduction}

We consider the connected graph $G=(V, E)$ with the cardinality of order $p$. Let $S=$ $\left\{a_{1}, a_{2}, a_{3}, \ldots, a_{p}\right\}$ be the $p$ number of non-negative integers. The thorn graph $G_{s}$ is acquired from the graph $G$ by joining $a_{i}$ number of pendent vertices to the vertex $v_{i}$ of $G$ for $i=$ $1,2, \ldots p$. These fixed $a_{i}$ pendent vertices to the vertices $v_{i}$ of $G$ are called thorns of $G$. Here we represent the set of $a_{i}$ number of pendent vertices to the vertex $v_{i}$ of $G$ by $V_{k}, \quad k=$ $1,2,3, \ldots, p$. Thus $V\left(G_{s}\right)=V(G) \cup V_{1}(G) \cup V_{2}(G) \cup V_{3}(G) \cup \ldots \cup V_{p}(G)$. In [1], Gutman et al., introduced the concept of thorn graphs. Along, with this, they established many applications related to chemistry $[1,2,5-7]$.

The topological $F_{i}$ the index was introduced to foresee the various temperature of the boiling point of hydrocarbons. There are two terms in the definition of $F_{i}$. in [2], Gutman et al., examined one of these terms, which is defined as follows.

Definition 1. Let $G=(V, E)$ be a connected graph and $d_{p}$ denotes the degree of the vertex $p$, then $R R^{-1}$ the index is defined as,

$$
R R^{-1}(G)=\sum_{p q \in E(G)} \sqrt{\left(d_{p}-1\right)\left(d_{q}-1\right)}
$$


In this paper, we discuss one of the currently established modified descriptions of Randic index, which is mentioned below.

Definition 2. Let $\mathrm{G}$ be the graph then the reciprocal randic index and is defined as

$$
R^{-1}(G)=\sum_{p q \in E(G)} \sqrt{d_{p} d_{q}}
$$

A topological index is a numerical value obtained mathematically in a distinct and very obvious manner from the given molecular graph. To design chemical compounds and its physio-chemical properties, it is mostly used in theoretical chemistry.

Definition 3. The topological index called the Atom-bond connectivity $(A B C)$ index was introduced by Estrada et al., [2]. This index is defined as follows [1, 2]

$$
A B C(G)=\sum_{p q \in E(G)} \sqrt{\frac{d_{p}+d_{q}-2}{d_{p} d_{q}}}
$$

Definition 4. For a molecular graph $G$, the geometric-arithmetic $(G A)$ index is defined as [6]

$$
G A(G)=\sum_{p q \in E(G)} \frac{2 \sqrt{d_{p} d_{q}}}{d_{p}+d_{q}}
$$

\section{Materials and Methods}

We collect all the information's and materials for our work digitally. The main focus of this paper is to obtain topological indices of standard thorn graphs and some of its other classes. Firstly, we identify the degree of each graph's vertices; later, we partition the edges of the graph, which follow the same property to procure our results.

\section{Results and Discussion}

In this portion of the paper, we found results related to the Reciprocal Randic index $\left(R^{-1}\right)$, Reduced Reciprocal Randic index $\left(R R^{-1}\right)$, Atom-bond Connectivity index $(A B C)$ and the geometric arithmetic index $(G A)$ of thorn graphs and its special cases.

3.1. Degree-based indices for standard graphs and some classes of its thorn graphs.

3.1.1. The complete graph $K_{a}$.

A complete graph $K_{a}$ is a graph in which every pair of vertices is adjacent. The graph $K_{a}$ has $a$ number of vertices with $\frac{a(a-1)}{2}$ number of edges.

\subsubsection{Thorn of the complete graph $K_{a}$.}

The b-thorn complete graph $K_{a, b}$ has a complete graph $K_{a}$ as the parent and $(b-a)$ thorns that is $u_{i}$ pendent vertices, $i=1,2, \ldots, b$ at each vertex $v_{i}$ for $i=1,2, \ldots, a$ of $K_{a}$ where $(a, b>2)$. The b-thorn complete graph $K_{a, b}$ is regarded as the thorn graph $\left(K_{a}\right)_{S}$ where $S=$ $\left(u_{1}, u_{2}, \ldots, u_{b}\right)$. Then the number of vertices of $K_{a, b}$, is $p=a+\sum_{i=1}^{b} \quad u_{i}$ and the number of edges are $q=\frac{n(n-1)}{2}+\sum_{i=1}^{b} \quad u_{i}$. The b-thorn complete graph $K_{a, b}$ is shown in Figure 1 . 


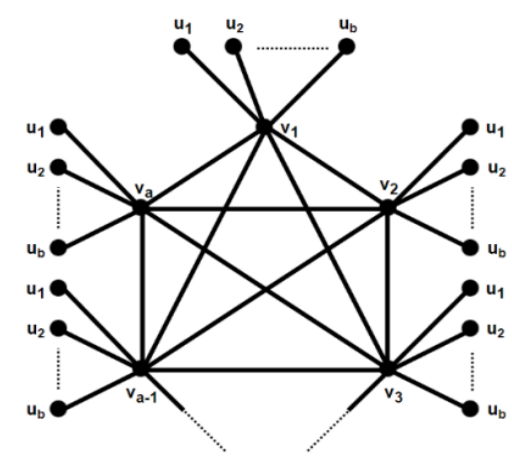

Figure 1. Thorn complete graph $K_{a, b}$.

Theorem 3.1. The reciprocal randic index of a thorn complete graph having $(a+b a)$ number of vertices is

$$
R^{-1}\left(K_{a, b}\right)=a_{i} b_{j} \sqrt{\left(b_{j}+a-1\right)}+a_{i}\left(b_{j}+a-1\right) .
$$

Proof: Here, we independently examine the pair of vertices of $K_{a}$ between the pair of vertices $u_{i}$ for $i=1,2,3, \ldots, b$ of $K_{a, b}$ along with the pair of vertices that belongs to $K_{a}$ and otherwise degree 1 vertex. Let $v_{1}, v_{2}, v_{3}, \ldots, v_{a}$ be the vertices of the complete graph $K_{a}$ where $i=2,3,4, \ldots, a$ and let $u_{1}, u_{2}, u_{3}, \ldots, u_{b}$ be a degree one vertices of $K_{a}$ where $j=1,2,3, \ldots, b$. (As in Figure 1). Let $d\left(v_{i}\right)=\left(b_{j}+a-1\right)$ and $d\left(u_{j}\right)=1$. Then we have,

$$
\begin{aligned}
R^{-1}\left(K_{a, b}\right) & =\sum_{i, j=1}^{a_{i}, b_{j}} \quad \sqrt{d\left(v_{i}\right) d\left(u_{j}\right)}+\sum_{i=1}^{a_{i}} \quad \sqrt{d\left(v_{i}\right) d\left(v_{i}\right)} \\
& =\sum_{i, j=1}^{a_{i}, b_{j}} \quad \sqrt{\left(b_{j}+a-1\right) 1}+\sum_{i=1}^{a_{i}} \quad \sqrt{\left(b_{j}+a-1\right)\left(b_{j}+a-1\right)} \\
& =a_{i} b_{j} \sqrt{\left(b_{j}+a-1\right)}+a_{i} \sqrt{\left(b_{j}+a-1\right)\left(b_{j}+a-1\right)} \\
& =a_{i} b_{j} \sqrt{\left(b_{j}+a-1\right)}+a_{i}\left(b_{j}+a-1\right) .
\end{aligned}
$$

Theorem 3.2: The reduced reciprocal randic index of a thorn complete graph having $(a+a b)$ number of vertices is

$$
R R^{-1}\left(K_{a, b}\right)=a_{i}\left(b_{j}+a-2\right) .
$$

Proof: Here we independently examine the pair of vertices of $K_{a}$ between the pair of vertices $u_{i}$ for $i=1,2,3, \ldots, b$ of $K_{a, b}$ along with the pair of vertices that belongs to $K_{a}$ and otherwise degree 1 vertex. Let $v_{1}, v_{2}, v_{3}, \ldots, v_{a}$ be the vertices of the complete graph $K_{a}$ where $i=2,3,4, \ldots, a$ and let $u_{1}, u_{2}, u_{3}, \ldots, u_{b}$ be a degree one vertices of $K_{a}$ where $j=1,2,3, \ldots, b$. (As in Figure 1). Let $d\left(v_{i}\right)=\left(b_{j}+a-1\right)$ and $d\left(u_{j}\right)=1$. Then we have,

$$
\begin{aligned}
& R R^{-1}\left(K_{a, b}\right)=\sum_{i, j=1}^{a_{i}, b_{j}} \quad \sqrt{\left(d\left(v_{i}\right)-1\right)\left(d\left(u_{j}\right)-1\right)}+\sum_{i=1}^{a_{i}} \quad \sqrt{\left(d\left(v_{i}\right)-1\right)\left(d\left(v_{i}\right)-1\right)} \\
& =\sum_{i, j=1}^{a_{i}, b_{j}} \quad \sqrt{\left(b_{j}+a-1\right)(1-1)}+ \\
& \sum_{i=1}^{a_{i}} \quad \sqrt{\left(b_{j}+a-1-1\right)\left(b_{j}+a-1-1\right)} \\
& =0+a_{i} \sqrt{\left(b_{j}+a-2\right)\left(b_{j}+a-2\right)} \\
& =a_{i}\left(b_{j}+a-2\right) \text {. }
\end{aligned}
$$

Theorem 3.3: The Atom-bond connectivity index of a thorn complete graph having $(a+$ $a b$ ) number of vertices is

$$
A B C\left(K_{a, b}\right)=a_{i} b_{j} \sqrt{\frac{\left(b_{j}+a-2\right)}{\left(b_{j}+a-1\right)}}+a_{i} \sqrt{\frac{2 b_{j}+2(a-2)}{\left(b_{j}+a-1\right)^{2}}}
$$


Proof: Here, we independently examine the pair of vertices of $K_{a}$ between the pair of vertices $u_{i}$ for $i=1,2,3, \ldots, b$ of $K_{a, b}$ along with the pair of vertices that belongs to $K_{a}$ and otherwise degree 1 vertex. Let $v_{1}, v_{2}, v_{3}, \ldots, v_{a}$ be the vertices of the complete graph $K_{a}$ where $i=2,3,4, \ldots, a$ and let $u_{1}, u_{2}, u_{3}, \ldots, u_{b}$ be a degree one vertices of $K_{a}$ where $j=$ $1,2,3, \ldots, b$. (As in Figure 1). Let $d\left(v_{i}\right)=\left(b_{j}+a-1\right)$ and $d\left(u_{j}\right)=1$. Then we have,

$$
\begin{aligned}
A B C\left(K_{a, b}\right) & =\sum_{i, j=1}^{a_{i}, b_{j}} \quad \sqrt{\frac{d\left(u_{j}\right)+d\left(v_{i}\right)-2}{d\left(u_{j}\right) \cdot d\left(v_{i}\right)}}+\sum_{i=1}^{a_{i}} \quad \sqrt{\frac{d\left(v_{i}\right)+d\left(v_{i}\right)-2}{d\left(v_{i}\right) \cdot d\left(v_{i}\right)}} \\
& =\sum_{i, j=1}^{a_{i}, b_{j}} \quad \sqrt{\frac{(1)+\left(b_{j}+a-1\right)-2}{(1)\left(b_{j}+a-1\right)}}+\sum_{i=1}^{a_{i}} \quad \sqrt{\frac{\left(b_{j}+a-1\right)+\left(b_{j}+a-1\right)-2}{\left(b_{j}+a-1\right)\left(b_{j}+a-1\right)}} \\
& =a_{i} b_{j} \sqrt{\frac{\left(b_{j}+a-2\right)}{\left(b_{j}+a-1\right)}}+a_{i} \sqrt{\frac{2 b_{j}+2(a-2)}{\left(b_{j}+a-1\right)^{2}}} .
\end{aligned}
$$

Theorem 3.4: The geometric-arithmetic index of a thorn complete graph having $(a+$ $a b$ )number of vertices is

$$
G A\left(K_{a, b}\right)=a_{i} b_{j} \frac{2 \sqrt{\left(b_{j}+a-1\right)}}{\left(b_{j}+a\right)}+a_{i} .
$$

Proof: Here, we independently examine the pair of vertices of $K_{a}$ between the pair of vertices $u_{i}$ for $i=1,2,3, \ldots, b$ of $K_{a, b}$ along with the pair of vertices that belongs to $K_{a}$ and otherwise degree 1 vertex. Let $v_{1}, v_{2}, v_{3}, \ldots, v_{a}$ be the vertices of the complete graph $K_{a}$ where $i=2,3,4, \ldots, a$ and let $u_{1}, u_{2}, u_{3}, \ldots, u_{b}$ be a degree one vertices of $K_{a}$ where $j=1,2,3, \ldots, b$. (As in Figure.1). Let $d\left(v_{i}\right)=\left(b_{j}+a-1\right)$ and $d\left(u_{j}\right)=1$. Then we have,

$$
\begin{array}{rlr}
G A\left(K_{a, b}\right) & =\sum_{i, j=1}^{a_{i}, b_{j}} \quad \frac{2 \sqrt{d\left(v_{i}\right) \cdot d\left(u_{j}\right)}}{d\left(v_{i}\right)+d\left(u_{j}\right)}+\sum_{i=1}^{a_{i}} & \frac{2 \sqrt{d\left(v_{i}\right) d\left(v_{i}\right)}}{d\left(v_{i}\right)+d\left(v_{i}\right)} \\
& =\sum_{i, j=1}^{a_{i}, b_{j}} \quad \frac{2 \sqrt{\left(b_{j}+a-1\right)}}{\left(b_{j}+a\right)}+\sum_{i=1}^{a_{i}} & \frac{2 \sqrt{\left(b_{j}+a-1\right)^{2}}}{\left(b_{j}+a-1+b_{j}+a-1\right)} \\
& =a_{i} b_{j} \frac{2 \sqrt{\left(b_{j}+a-1\right)}}{\left(b_{j}+a\right)}+a_{i} .
\end{array}
$$

\subsubsection{A cog-complete graph $K^{c}{ }_{a}$.}

$K_{a}^{c}$ is the graph formed from a complete graph $K_{a}(a \geq 2)$ of the vertex set $\left\{v_{1}, v_{2}, v_{3}, \ldots, v_{a}\right\}$ with the addition of $b$ number of vertices such as $\left\{u_{1}, u_{2}, u_{3}, \ldots \ldots, u_{b}\right\}$ and $2 b$ number of edges given by $\left\{u_{j} v_{i}, u_{j} v_{i+1}: i=1,2,3, \ldots, a\right.$ and $\left.j=1,2,3, \ldots, b\right\}\left(v_{a+1}=\right.$ $\left.v_{1}\right)$, as shown in Figure 2.

We know that $p\left(K_{a}^{c}\right)=2(a+b), q\left(K_{a}^{c}\right)=\frac{a(a+3)}{2}$.

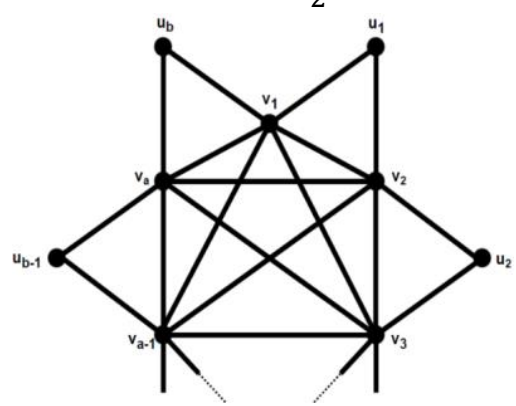

Figure 2. Cog-Complete graph $K^{c}{ }_{a}$.

3.1.4. A thorn cog-complete graph $K^{c^{*}} a$ 
$K^{c^{*}}{ }_{a}$ is the cog-complete graph $K^{c}{ }_{a}(a \geq 2)$ is obtained from Definition 3., with $2 b$ number of additional vertices of degree 1 such as $\left\{w_{1}, w_{2}, w_{3}, \ldots ., w_{2 b}\right\}$ for $k=$ $1,2,3, \ldots \ldots, 2 b$ and edges given by $\left\{u_{j} w_{2 k-1}, u_{j} w_{2 k}: i, j=1,2,3, \ldots \ldots, b\right\}$, as shown in Figure 3 .

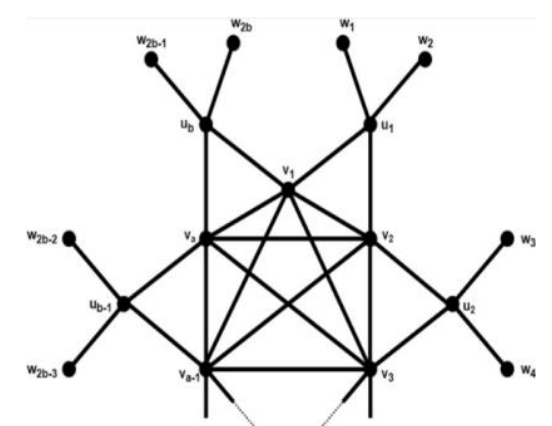

Figure 3. Thorn Cog-Complete graph $K^{c^{*}}{ }_{b, a}$.

Theorem 3.5: The reciprocal randic index of a thorn cog-complete graph having $(a+3 b)$ number of vertices is

$$
R^{-1}\left(K_{b, a}^{c^{*}}\right)=2 a_{i} b_{j} \sqrt{(a+1)}+a_{i}(a+1)+4 b_{j} b_{k} .
$$

Proof: Here, we independently examine the pair of vertices of $K_{a}$ between the pair of vertices $u_{i}$ for $i=1,2,3, \ldots, b$ of $K_{a}^{c}$ along with the pair of vertices that belongs to $K_{a}$ and between the pair of vertices $u_{i}$, for $i=1,2,3, \ldots, b$ of $K^{c}{ }_{a}$ along with the pair of vertices degree 1 vertices of $K^{c^{*}}{ }_{b, a}$. Let $v_{1}, v_{2}, v_{3}, \ldots, v_{a}$ be the vertices of the complete graph $K_{a}$ where $i=$ $2,3,4, \ldots, a$ and let $u_{1}, u_{2}, u_{3}, \ldots, u_{b}$ be the addition of $b$ number of vertices of $K^{c}{ }_{a}$ where $j=$ $1,2,3, \ldots, b$. Also, let $w_{1}, w_{2}, \ldots \ldots, w_{2 b}, k=1,2,3, \ldots, 2 b$ of $K^{c^{*}}{ }_{b, a}$ be an additional $2 b$ number of degree 1 vertices of $K^{c^{*}}{ }_{b, a}$. (As in Figure. 3). Let $d\left(v_{i}\right)=(a+1), d\left(u_{j}\right)=4$ and $d\left(w_{k}\right)=1$. Then we have,

$$
\begin{aligned}
& R^{-1}\left(K^{c^{*}}{ }_{b, a}\right)=\sum_{i, j=1}^{a_{i}, b_{j}} \quad \sqrt{d\left(v_{i}\right) d\left(u_{j}\right)}+\sum_{i=1}^{a_{i}} \quad \sqrt{d\left(v_{i}\right) d\left(v_{i}\right)}+\sum_{j, k=1}^{b_{j}, 2 b_{k}} \quad \sqrt{d\left(u_{j}\right) d\left(w_{k}\right)} \\
& =\sum_{i, j=1}^{a_{i}, b_{j}} \quad \sqrt{(a+1) 4}+\sum_{i=1}^{a_{i}} \quad \sqrt{(a+1)(a+1)}+\sum_{j, k=1}^{b_{j}, 2 b_{k}} \quad \sqrt{1(4)} \\
& =a_{i} b_{j} \sqrt{4(a+1)}+a_{i} \sqrt{(a+1)(a+1)}+b_{j}\left(2 b_{k}\right) 2 \\
& =2 a_{i} b_{j} \sqrt{(a+1)}+a_{i}(a+1)+4 b_{j} b_{k} \text {. }
\end{aligned}
$$

Theorem 3.6: The reduced reciprocal randic index of a thorn cog-complete graph having $(a+3 b)$ number of vertices is

$$
R R^{-1}\left(K^{c^{*}}{ }_{b, a}\right)=a_{i} b_{j} \sqrt{3 a}+a_{i}(a) .
$$

Proof: Here, we independently examine the pair of vertices of $K_{a}$ between the pair of vertices $u_{i}$ for $i=1,2,3, \ldots, b$ of $K^{c}{ }_{a}$ along with the pair of vertices that belongs to $K_{a}$ and between the pair of vertices $u_{i}$ for $i=1,2,3, \ldots, b$ of $K^{c}{ }_{a}$ along with the pair of vertices degree 1 vertices of $K^{c^{*}}{ }_{b, a}$. Let $v_{1}, v_{2}, v_{3}, \ldots, v_{a}$ be the vertices of the complete graph $K_{a}$ where $i=$ $2,3,4, \ldots, a$ and let $u_{1}, u_{2}, u_{3}, \ldots, u_{b}$ be the addition of $b$ number of vertices of $K^{c}{ }_{a}$ where $j=$ $1,2,3, \ldots, b$. Also, let $w_{1}, w_{2}, \ldots \ldots, w_{2 b}, k=1,2,3, \ldots, 2 b$ of $K^{c^{*}}{ }_{b, a}$ be an additional $2 b$ number of degree 1 vertices of $K^{c^{*}}{ }_{b, a}$. (As in Figure. 3). Let $d\left(v_{i}\right)=(a+1), d\left(u_{j}\right)=4$ and $d\left(w_{k}\right)=1$. Then we have, 


$$
\begin{aligned}
& R R^{-1}\left(K_{b, a}^{c^{*}}\right)=\sum_{i, j=1}^{a_{i}, b_{j}} \sqrt{\left(d\left(v_{i}\right)-1\right)\left(d\left(u_{j}\right)-1\right)}+\sum_{i=1}^{a_{i}} \sqrt{\left(d\left(v_{i}\right)-1\right)^{2}} \\
& +\sum_{j, k=1}^{b_{j}, 2 b_{k}} \sqrt{\left(d\left(u_{j}\right)-1\right)\left(d\left(w_{k}\right)-1\right)} \\
& = \\
& \sum_{i=1}^{a_{i}} \quad \sqrt{(a+1-1)(a+1-1)} \\
& \sum_{i, j=1}^{a_{i}, b_{j}} \quad \sqrt{(a+1-1)(4-1)}+ \\
& +\sum_{j, k=1}^{b_{j, 2 b_{k}}} \quad \sqrt{(1-1)(4-1)} \\
& =a_{i} b_{j} \sqrt{3 a}+a_{i} \sqrt{a(a)} \\
& =a_{i} b_{j} \sqrt{3 a}+a_{i}(a) \text {. }
\end{aligned}
$$

Theorem 3.7: The Atom-bond connectivity index of a thorn cog-complete graph having $(a+3 b)$ number of vertices is

$$
A B C\left(K_{b, a}^{c^{*}}\right)=a_{i} b_{j} \sqrt{\frac{a+3}{4(a+1)}}+a_{i} \frac{\sqrt{2 a}}{(a+1)}+b_{j} b_{k} \sqrt{3} .
$$

Proof: Here, we independently examine the pair of vertices of $K_{a}$ between the pair of vertices $u_{i}$ for $i=1,2,3, \ldots, b$ of $K^{c}{ }_{a}$ along with the pair of vertices that belongs to $K_{a}$ and between the pair of vertices $u_{i}$ for $i=1,2,3, \ldots, b$ of $K_{a}^{c}$ along with the pair of vertices degree 1 vertices of $K^{c^{*}}{ }_{b, a}$. Let $v_{1}, v_{2}, v_{3}, \ldots, v_{a}$ be the vertices of the complete graph $K_{a}$ where $i=$ $2,3,4, \ldots, a$ and let $u_{1}, u_{2}, u_{3}, \ldots, u_{b}$ be the addition of $b$ number of vertices of $K^{c}{ }_{a}$ where $j=$ $1,2,3, \ldots, b$. Also, let $w_{1}, w_{2}, \ldots \ldots, w_{2 b}, k=1,2,3, \ldots, 2 b$ of $K^{c^{*}}{ }_{b, a}$, be an additional $2 b$ number of degree 1 vertices of $K^{c^{*}}{ }_{b, a}$. (As in Figure. 3). Let $d\left(v_{i}\right)=(a+1), d\left(u_{j}\right)=4$ and $d\left(w_{k}\right)=1$. Then we have,

$$
\begin{aligned}
A B C\left(K^{c^{*}}{ }_{b, a}\right) & =\sum_{i, j=1}^{a_{i}, b_{j}} \quad \sqrt{\frac{d\left(v_{i}\right)+d\left(u_{j}\right)-2}{d\left(v_{i}\right) d\left(u_{j}\right)}}+\sum_{i=1}^{a_{i}} \quad \sqrt{\frac{d\left(v_{i}\right)+d\left(v_{i}\right)-2}{d\left(v_{i}\right) d\left(v_{i}\right)}}+\sum_{j, k=1}^{b_{j}, 2 b_{k}} \quad \sqrt{\frac{d\left(u_{j}\right)+d\left(w_{k}\right)-2}{d\left(u_{j}\right) d\left(w_{k}\right)}} \\
& =\sum_{i, j=1}^{a_{i}, b_{j}} \quad \sqrt{\frac{a+1+4-2}{4(a+1)}}+\sum_{i=1}^{a_{i}} \sqrt{\frac{(a+1)+(a+1)-2}{(a+1)^{2}}+\sum_{j, k=1}^{b_{j}, 2 b_{k}} \quad \sqrt{\frac{4+1-2}{(4) 1}}} \\
& =a_{i} b_{j} \sqrt{\frac{a+3}{4(a+1)}}+a_{i} \sqrt{\frac{2 a}{(a+1)^{2}}}+b_{j}\left(2 b_{k}\right) \sqrt{\frac{3}{(4)}} \\
& =a_{i} b_{j} \sqrt{\frac{a+3}{4(a+1)}}+a_{i} \frac{\sqrt{2 a}}{(a+1)}+b_{j} b_{k} \sqrt{3}
\end{aligned}
$$

Theorem 3.8: The geometric-arithmetic index of a thorn cog-complete graph having $(a+3 b)$ number of vertices is

$$
G A\left(K_{b, a}^{c^{*}}\right)=a_{i} b_{j} \frac{4 \sqrt{(a+1)}}{(a+5)}+a_{i}+\frac{8 b_{j} b_{k}}{5} .
$$

Proof: Here we independently examine the pair of vertices of $K_{a}$ between the pair of vertices $u_{i}$ for $i=1,2,3, \ldots, b$ of $K^{c}{ }_{a}$ along with the pair of vertices which belongs to $K_{a}$ and between the pair of vertices $u_{i}$ for $i=1,2,3, \ldots, b$ of $K^{c}{ }_{a}$ along with the pair of vertices degree 1 vertices of $K^{c^{*}}{ }_{b, a}$. Let $v_{1}, v_{2}, v_{3}, \ldots, v_{a}$ be the vertices of the complete graph $K_{a}$ where $i=$ 
$2,3,4, \ldots, a$ and let $u_{1}, u_{2}, u_{3}, \ldots, u_{b}$ be addition of $b$ number of vertices of $K_{a}^{c}$ where $j=$ $1,2,3, \ldots, b$. Also, let $w_{1}, w_{2}, \ldots \ldots, w_{2 b}, k=1,2,3, \ldots, 2 b$ of $K^{c^{*}}{ }_{b, a}$ be an additional $2 b$ number of degree 1 vertices of $K^{c^{*}}{ }_{b, a}$. (As in Figure. 3). Let $d\left(v_{i}\right)=(a+1), d\left(u_{j}\right)=4$ and $d\left(w_{k}\right)=1$. Then we have,

$$
\begin{aligned}
& G A\left(K_{b, a}^{c^{*}}\right)=\sum_{i, j=1}^{a_{i}, b_{j}} \quad \frac{2 \sqrt{d\left(v_{i}\right) d\left(u_{j}\right)}}{d\left(v_{i}\right)+d\left(u_{j}\right)}+\sum_{i=1}^{a_{i}} \quad \frac{2 \sqrt{d\left(v_{i}\right) d\left(v_{i}\right)}}{d\left(v_{i}\right)+d\left(v_{i}\right)}+\sum_{j, k=1}^{b_{j}, 2 b_{k}} \quad \frac{2 \sqrt{d\left(u_{j}\right) d\left(w_{k}\right)}}{d\left(u_{j}\right)+d\left(w_{k}\right)} \\
& =\sum_{i, j=1}^{a_{i}, b_{j}} \quad \frac{2 \sqrt{4(a+1)}}{(a+1+4)}+\sum_{i=1}^{a_{i}} \quad \frac{2 \sqrt{(a+1)(a+1)}}{(a+1)+(a+1)}+\sum_{j, k=1}^{b_{j}, 2 b_{k}} \quad \frac{2 \sqrt{4(1)}}{(5)} \\
& =a_{i} b_{j} \frac{4 \sqrt{(a+1)}}{a+5}+a_{i} \frac{2 \sqrt{(a+1)^{2}}}{2 a+2}+b_{j}\left(2 b_{k}\right) \frac{4}{5} \\
& =a_{i} b_{j} \frac{4 \sqrt{(a+1)}}{a+5}+a_{i}+\frac{8 b_{j} b_{k}}{5} .
\end{aligned}
$$

\subsubsection{A cog-star graph $S^{c}{ }_{a}$.}

$S^{c}{ }_{a}$ is the graph formed from a star graph $S_{a}(a \geq 4)$, of the vertex set $\left\{v_{1}, v_{2}, v_{3}, \ldots, v_{a-1}, v_{a}\right\}$ with the addition of $(b-1)$ number of vertices such as $\left\{u_{1}, u_{2}, u_{3}, \ldots, u_{b-1}\right\}$ and $2 b$ number of edges given by $\left\{u_{j} v_{i+1}, u_{j} v_{i+2}: i=\right.$ $1,2,3, \ldots, a$ and $j=1,2,3, \ldots \ldots,(b-1)\}\left(v_{a+1}=v_{2}\right)$, as shown in Figure 4 .

We know that $p\left(S^{c}{ }_{a}\right)=(a+b-1), q\left(S_{a}^{c}\right)=a+2 b-3$.

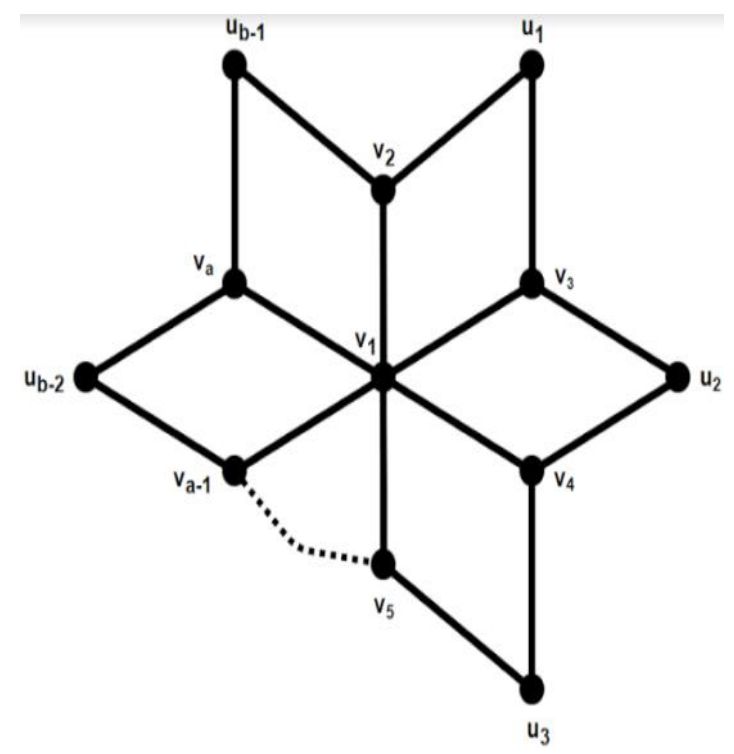

Figure 4. A Cog-Star graph $S_{a}^{c}$.

3.1.6. The thorn Cog-Star graph $S^{c^{*}} a$.

$S^{c^{*}}$ is the graph formed from a star graph $S_{a}^{c}(a \geq 4)$, of the vertex set $\left\{v_{1}, v_{2}, v_{3}, \ldots, v_{a-1}, v_{a}, u_{1}, u_{2}, u_{3}, \ldots ., u_{b-1}\right\}$ for $i=1,2,3, \ldots$, and $j=1,2,3, \ldots \ldots,(b-$ 1) with the addition of $2(b-1)$ number of vertices such as $\left\{w_{1}, w_{2}, w_{3}, \ldots \ldots, w_{2 b-3}, w_{2 b-2}\right\}$ and edges given by $\left\{u_{j} w_{2 j-1}, u_{j} w_{2 j}: j=1,2,3, \ldots \ldots,(b-1)\right\}$, as shown in Figure 5 . 


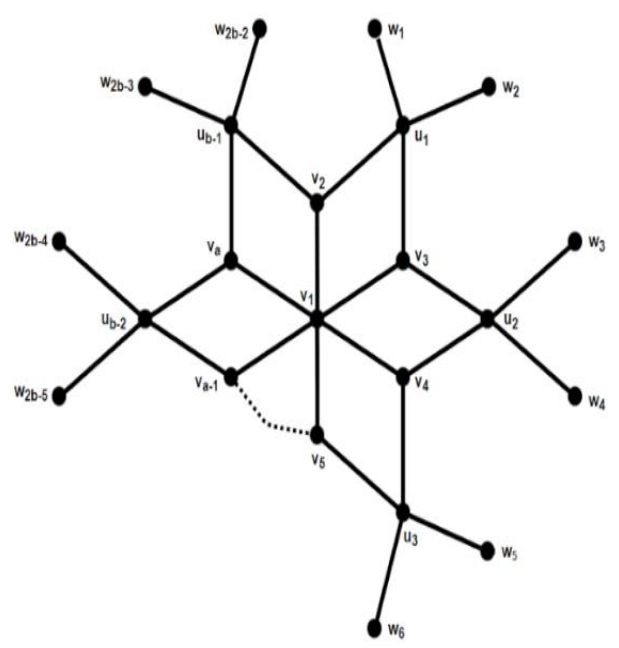

Figure 5. Thorn Cog-Star graph $S^{c^{*}} a$.

Theorem 3.9: The reciprocal randic index of a thorn cog-star graph having $(a+3(b-1))$ number of vertices is

$$
R^{-1}\left(S^{c^{*}} a\right)=2 a_{i}\left(b_{j}-1\right) \sqrt{3}+a_{i} \sqrt{3(a-1)}+4\left(b_{j}-1\right)\left(b_{k}-1\right) .
$$

Proof: Here, we independently examine the pair of vertices of $S_{a}$ between the pair of vertices $u_{j}$ for $j=1,2,3, \ldots,(b-1)$ of $S^{c}{ }_{a}$ along with the pair of vertices that belongs to $S_{a}$ and between the pair of vertices $u_{j}$ for $j=1,2,3, \ldots,(b-1)$ of $S^{c}{ }_{a}$ along with the pair of vertices degree 1 vertices of $S^{c^{*}}{ }_{a}$. Let $v_{1}, v_{2}, v_{3}, \ldots, v_{a}$ be the vertices of a star graph $S_{a}$ where $i$ ranges from $1,2,3,4, \ldots, a$ and let $u_{1}, u_{2}, u_{3}, \ldots, u_{b-1}$ be the addition of $(b-1)$ number of vertices of $S_{a}^{c}$ where $j=1,2,3, \ldots,(b-1)$. Also, let $w_{1}, w_{2}, \ldots \ldots, w_{2 b-2}, k=$ $1,2,3, \ldots,(2 b-2)$ of $S^{c^{*}} a$ be an additional $(2 b-2)$ number of degree 1 vertices of $S^{c^{*}}{ }_{a}$. (As in Figure. 5). Let $d\left(v_{1}\right)=(a-1), d\left(u_{j}\right)=4, d\left(v_{i}\right)=3, i \in\{2,3,4, \ldots \ldots, a\}$ and $d\left(w_{k}\right)=1$. Then we have,

$$
\begin{aligned}
R^{-1}\left(S^{c^{*}}\right) & =\sum_{i=2, j=1}^{a_{i,}\left(b_{j}-1\right)} \sqrt{d\left(v_{i}\right) d\left(u_{j}\right)}+\sum_{i=2}^{a_{i}} \quad \sqrt{d\left(v_{1}\right) d\left(v_{i}\right)} \\
& +\sum_{j, k=1}^{b_{j}-1,2 b_{k}-2} \quad \sqrt{d\left(u_{j}\right) d\left(w_{k}\right)} \\
& =\sum_{i=2, j=1}^{a_{i,}\left(b_{j}-1\right)} \sqrt{(3) 4}+\sum_{i=2}^{a_{i}} \quad \sqrt{(a-1) 3}+\sum_{j, k=1}^{b_{j}-1,2 b_{k}-2} \quad \sqrt{1(4)} \\
& =a_{i}\left(b_{j}-1\right) \sqrt{4(3)}+a_{i} \sqrt{(a-1)(3)}+\left(b_{j}-1\right)\left(2 b_{k}-2\right) 2 \\
& =2 a_{i}\left(b_{j}-1\right) \sqrt{3}+a_{i} \sqrt{3(a-1)}+4\left(b_{j}-1\right)\left(b_{k}-1\right) .
\end{aligned}
$$

Theorem 3.10: The reduced reciprocal randic index of a thorn cog-star graph having $(a+3(b-1))$ number of vertices is

$$
R R^{-1}\left(S_{a}^{c^{*}}\right)=a_{i}\left(b_{j}-1\right) \sqrt{6}+a_{i} \sqrt{2(a-2)} .
$$

Proof: Here, we independently examine the pair of vertices of $S_{a}$ between the pair of vertices $u_{j}$ for $j=1,2,3, \ldots,(b-1)$ of $S^{c}{ }_{a}$ along with the pair of vertices that belongs to $S_{a}$ and between the pair of vertices $u_{j}$ for $j=1,2,3, \ldots,(b-1)$ of $S^{c}{ }_{a}$ along with the pair of 
vertices degree 1 vertices of $S^{c^{*}}$. Let $v_{1}, v_{2}, v_{3}, \ldots, v_{a}$ be the vertices of a star graph $S_{a}$ where $i$ ranges from $1,2,3,4, \ldots, a$ and let $u_{1}, u_{2}, u_{3}, \ldots, u_{b-1}$ be the addition of $(b-1)$ number of vertices of $S^{c}{ }_{a}$ where $j=1,2,3, \ldots,(b-1)$. Also, let $w_{1}, w_{2}, \ldots \ldots, w_{2 b-2}, k=$ $1,2,3, \ldots,(2 b-2)$ of $S^{c^{*}} a$ be an additional $(2 b-2)$ number of degree 1 vertices of $S^{c^{*}}{ }_{a}$. (As in Figure. 5). Let $d\left(v_{1}\right)=(a-1), d\left(u_{j}\right)=4, d\left(v_{i}\right)=3, i \in\{2,3,4, \ldots \ldots, a\}$ and $d\left(w_{k}\right)=1$. Then we have,

$$
\begin{aligned}
& R R^{-1}\left(S^{c^{*}}{ }_{a}\right)=\quad \sum_{i=2, j=1}^{a_{i,}\left(b_{j}-1\right)} \sqrt{\left(d\left(v_{i}\right)-1\right)\left(d\left(u_{j}\right)-1\right)}+ \\
& \sum_{i=2}^{a_{i}} \quad \sqrt{\left(d\left(v_{1}\right)-1\right)\left(d\left(v_{i}\right)-1\right)}+ \\
& \sum_{j, k=1}^{b_{j}-1,2 b_{k}-2} \sqrt{\left(d\left(u_{j}\right)-1\right)\left(d\left(w_{k}\right)-1\right)} \\
& =\sum_{i=2, j=1}^{a_{i,}\left(b_{j}-1\right)} \quad \sqrt{(3-1)(4-1)}+\sum_{i=2}^{a_{i}} \quad \sqrt{(a-1-1)(3-1)} \\
& +\sum_{j, k=1}^{b_{j}-1,2 b_{k}-2} \sqrt{(1-1)(4-1)} \\
& =a_{i}\left(b_{j}-1\right) \sqrt{6}+a_{i} \sqrt{2(a-2)} \text {. }
\end{aligned}
$$

Theorem 3.11: The Atom-bond connectivity index of a thorn cog-star graph having $(a+3(b-1))$ number of vertices is

$$
A B C\left(S_{a}^{c^{*}}\right)=a_{i}\left(b_{j}-1\right) \sqrt{\frac{5}{12}}+a_{i} \sqrt{\frac{a}{3(a-1)}}+\left(b_{j}-1\right)\left(b_{k}-1\right) \sqrt{3} .
$$

Proof: Here, we independently examine the pair of vertices of $S_{a}$ between the pair of vertices $u_{j}$ for $j=1,2,3, \ldots,(b-1)$ of $S^{c}{ }_{a}$ along with the pair of vertices that belongs to $S_{a}$ and between the pair of vertices $u_{j}$ for $j=1,2,3, \ldots,(b-1)$ of $S^{c}{ }_{a}$ along with the pair of vertices degree 1 vertices of $S^{c^{*}}{ }_{a}$. Let $v_{1}, v_{2}, v_{3}, \ldots, v_{a}$ be the vertices of a star graph $S_{a}$ where $i$ ranges from $1,2,3,4, \ldots, a$ and let $u_{1}, u_{2}, u_{3}, \ldots, u_{b-1}$ be the addition of $(b-1)$ number of vertices of $S^{c}{ }_{a}$ where $j=1,2,3, \ldots,(b-1)$. Also, let $w_{1}, w_{2}, \ldots \ldots, w_{2 b-2}, k=$ $1,2,3, \ldots,(2 b-2)$ of $S^{c^{*}} a$ be an additional $(2 b-2)$ number of degree 1 vertices of $S^{c^{*}}{ }_{a}$. (As in Figure. 5). Let $d\left(v_{1}\right)=(a-1), d\left(u_{j}\right)=4, d\left(v_{i}\right)=3, i \in\{2,3,4, \ldots \ldots, a\}$ and $d\left(w_{k}\right)=1$. Then we have,

$$
\begin{gathered}
A B C\left(S^{c^{*}}\right)=\sum_{i=2, j=1}^{a_{i,}\left(b_{j}-1\right)} \quad \sqrt{\frac{d\left(v_{i}\right)+d\left(u_{j}\right)-2}{d\left(v_{i}\right) d\left(u_{j}\right)}}+ \\
\sum_{i=2}^{a_{i}} \sqrt{\frac{d\left(v_{1}\right)+d\left(v_{i}\right)-2}{d\left(v_{1}\right) d\left(v_{i}\right)}+\sum_{j, k=1}^{b_{j}-1,2 b_{k}-2} \sqrt{\frac{d\left(u_{j}\right)+d\left(w_{k}\right)-2}{d\left(u_{j}\right) d\left(w_{k}\right)}}} \\
=\sum_{i=2, j=1}^{a_{i,},\left(b_{j}-1\right)} \sqrt{\frac{3+4-2}{3(4)}}+\sum_{i=2}^{a_{i}} \quad \sqrt{\frac{(a-1)+(3)-2}{3(a-1)}+\sum_{j, k=1}^{b_{j}-1,2 b_{k}-2}} \sqrt{\frac{4+1-2}{(4)(1)}} \\
=a_{i}\left(b_{j}-1\right) \sqrt{\frac{5}{4(3)}}+a_{i} \sqrt{\frac{a}{3(a-1)}}+\left(b_{j}-1\right)\left(2 b_{k}-2\right) \sqrt{\frac{3}{(4)}}
\end{gathered}
$$




$$
=a_{i}\left(b_{j}-1\right) \sqrt{\frac{5}{12}}+a_{i} \sqrt{\frac{a}{3(a-1)}}+\left(b_{j}-1\right)\left(b_{k}-1\right) \sqrt{3} .
$$

Theorem 3.12: The geometric-arithmetic index of a thorn cog-star graph having $(a+$ $3(b-1))$ number of vertices is

$G A\left(S_{a}^{c^{*}}\right)=a_{i}\left(b_{j}-1\right) \frac{4 \sqrt{3}}{7}+a_{i} \frac{2 \sqrt{3(a-1)}}{(a+2)}+\frac{8\left(b_{j}-1\right)\left(b_{k}-1\right)}{5}$.

Proof: Here, we independently examine the pair of vertices of $S_{a}$ between the pair of vertices $u_{j}$ for $j=1,2,3, \ldots,(b-1)$ of $S^{c}{ }_{a}$ along with the pair of vertices that belongs to $S_{a}$ and between the pair of vertices $u_{j}$ for $j=1,2,3, \ldots,(b-1)$ of $S^{c}{ }_{a}$ along with the pair of vertices degree 1 vertices of $S^{c^{*}}$. Let $v_{1}, v_{2}, v_{3}, \ldots, v_{a}$ be the vertices of a star graph $S_{a}$ where $i$ ranges from $1,2,3,4, \ldots, a$ and let $u_{1}, u_{2}, u_{3}, \ldots, u_{b-1}$ be the addition of $(b-1)$ number of vertices of $S^{c}{ }_{a}$ where $j=1,2,3, \ldots,(b-1)$. Also, let $w_{1}, w_{2}, \ldots \ldots, w_{2 b-2}, k=$ $1,2,3, \ldots,(2 b-2)$ of $S^{c^{*}}{ }_{a}$ be an additional $(2 b-2)$ number of degree 1 vertices of $S^{c^{*}} a$. (As in Figure. 5). Let $d\left(v_{1}\right)=(a-1), d\left(u_{j}\right)=4, d\left(v_{i}\right)=3, i=2,3,4, \ldots \ldots, a$ and $d\left(w_{k}\right)=1$. Then we have,

$$
\begin{aligned}
G A\left(S_{a}^{c^{*}}\right)= & \sum_{i=2, j=1}^{a_{i},\left(b_{j}-1\right)} \quad \frac{2 \sqrt{d\left(v_{i}\right) d\left(u_{j}\right)}}{\left(d\left(v_{i}\right)+d\left(u_{j}\right)\right)}+\sum_{i=2}^{a_{i}} \quad \frac{2 \sqrt{d\left(v_{1}\right) d\left(v_{i}\right)}}{\left(d\left(v_{1}\right)+d\left(v_{i}\right)\right)} \\
& +\sum_{j, k=1}^{b_{j}-1,2 b_{k}-2} \quad \frac{2 \sqrt{d\left(u_{j}\right) d\left(w_{k}\right)}}{\left(d\left(u_{j}\right)+d\left(w_{k}\right)\right)} \\
= & \sum_{i=2, j=1}^{\left.a_{i,}, b_{j}-1\right)} \quad \frac{2 \sqrt{3(4)}}{(3+4)}+\sum_{i=2}^{a_{i}} \quad \frac{2 \sqrt{3(a-1)}}{((a-1)+3)}+\sum_{j, k=1}^{b_{j}-1,2 b_{k}-2} \quad \frac{2 \sqrt{1(4)}}{(4+1)} \\
= & a_{i}\left(b_{j}-1\right) \frac{4 \sqrt{3}}{7}+a_{i} \frac{2 \sqrt{3(a-1)}}{(a+2)}+\left(b_{j}-1\right)\left(2 b_{k}-2\right) \frac{4}{5} \\
= & a_{i}\left(b_{j}-1\right) \frac{4 \sqrt{(3)}}{(7)}+a_{i} \frac{2 \sqrt{3(a-1)}}{(a+2)}+\frac{8\left(b_{j}-1\right)\left(b_{k}-1\right)}{5} .
\end{aligned}
$$

3.1.7. Thorn of wheel graph $W_{a}$.

The b-thorn wheel graph $W_{a, b}$ has a wheel graph $W_{a}$ as the parent, and $(b-3)$ thorns that are $u_{i}$ pendent vertices $i=1,2, \ldots, b$, at each vertex $v_{i}$ for $i=1,2, \ldots, a$ of $W_{a}$ where $a, b>3$. The b-thorn wheel graph $W_{a, b}$ is regarded as the thorn graph $\left(W_{a}\right)_{S}$ where $S=$ $\left(u_{1}, u_{2}, \ldots, u_{b}\right)$. Then the number of vertices of $W_{a, b}$ is $p=a+\sum_{i=1}^{b} \quad u_{i}$ and the number of edges are $q=2(a-1)+\sum_{i=1}^{b} \quad u_{i}$. The b-thorn wheel graph $W_{a, b}$ is shown in Figure 6 . 


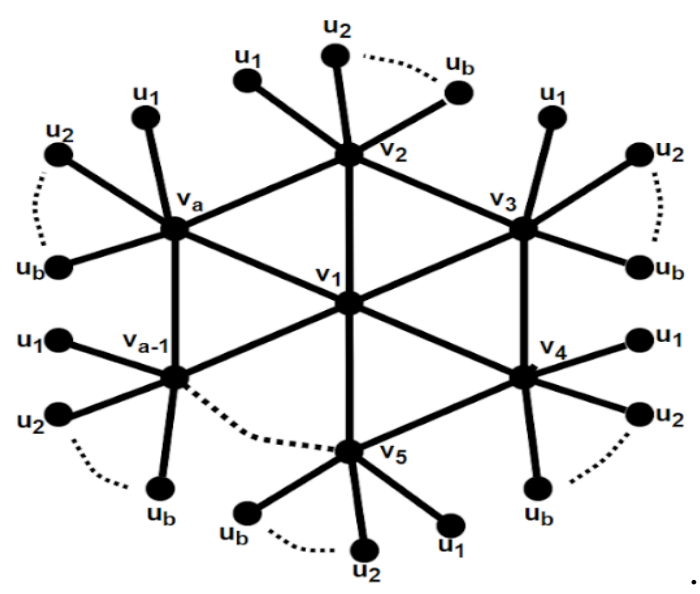

Figure 6. Thorn Wheel graph $W_{a, b}$.

Theorem 3.13: The reciprocal randic index of a thorn wheel graph having $(a+$ $(a-1) b)$ number of vertices is

$$
R^{-1}\left(W_{a, b}\right)=a_{i} b_{j} \sqrt{(b+3)}+a_{i}(b+3)+a_{i} \sqrt{(a-1)(b+3)}
$$

Proof: Here, we independently examine the pair of vertices of $W_{a}$ between the pair of vertices $u_{i}$ for $i=1,2,3, \ldots, b$ of $W_{a, b}$ along with the pair of vertices that belongs to $W_{a}$ and otherwise degree 1 vertex. Let $v_{1}, v_{2}, v_{3}, \ldots, v_{a}$ be the vertices of wheel graph $W_{a}$ where i ranges from $1,2,3,4, \ldots, a$ and let $u_{1}, u_{2}, u_{3}, \ldots, u_{b}$ be a degree one vertices of $W_{a, b}$ where $j=1,2,3, \ldots, b$. (As in Figure. 6). Let $d\left(v_{i}\right)=(b+3)$ for $i=2,3,4, \ldots . a, d\left(v_{1}\right)=$ $(a-1)$ and $d\left(u_{j}\right)=1$. Then we have,

$$
\begin{aligned}
R^{-1}\left(W_{a, b}\right)= & \sum_{i=2, j=1}^{a_{i}, b_{j}} \sqrt{d\left(v_{i}\right) d\left(u_{j}\right)}+\sum_{i=2}^{a_{i}} \sqrt{d\left(v_{i}\right) d\left(v_{i}\right)} \\
& +\sum_{i=2}^{a_{i}} \sqrt{d\left(v_{1}\right) d\left(v_{i}\right)} \\
= & \sum_{i=2, j=1}^{a_{i}, b_{j}} \sqrt{(b+3) 1}+\sum_{i=2}^{a_{i}} \sqrt{(b+3)(b+3)} \\
& +\sum_{i=2}^{a_{i}} \sqrt{(a-1)(b+3)} \\
= & a_{i} b_{j} \sqrt{(b+3)}+a_{i} \sqrt{(b+3)^{2}}+a_{i} \sqrt{(a-1)(b+3)} \\
= & a_{i} b_{j} \sqrt{(b+3)}+a_{i}(b+3)+a_{i} \sqrt{(a-1)(b+3)}
\end{aligned}
$$

Theorem 3.14: The reduced reciprocal randic index of a thorn wheel graph having $(a+$ $(a-1) b)$ number of vertices is

$$
R R^{-1}\left(W_{a, b}\right)=a_{i}(b+2)+a_{i} \sqrt{(a-2)(b+2)} .
$$

Proof: Here, we independently examine the pair of vertices of $W_{a}$ between the pair of vertices $u_{i}$ for $i=1,2,3, \ldots, b$ of $W_{a, b}$ along with the pair of vertices that belongs to $W_{a}$ and 
otherwise degree 1 vertex. Let $v_{1}, v_{2}, v_{3}, \ldots, v_{a}$ be the vertices of wheel graph $W_{a}$ where i ranges from $1,2,3,4, \ldots, a$ and let $u_{1}, u_{2}, u_{3}, \ldots, u_{b}$ be a degree one vertices of $W_{a, b}$ where $j=1,2,3, \ldots, b$. (As in Figure. 6). Let $d\left(v_{i}\right)=(b+3)$ for $i=2,3,4, \ldots . a, d\left(v_{1}\right)=$ $(a-1)$ and $d\left(u_{j}\right)=1$. Then we have,

$$
\begin{gathered}
R R^{-1}\left(W_{a, b}\right)=\sum_{i=2, j=1}^{a_{i}, b_{j}} \sqrt{\left(d\left(v_{i}\right)-1\right)\left(d\left(u_{j}\right)-1\right)}+\sum_{i=2}^{a_{i}} \sqrt{\left(d\left(v_{i}\right)-1\right)^{2}} \\
+\sum_{i=2}^{a_{i}} \sqrt{\left(d\left(v_{1}\right)-1\right)\left(d\left(v_{i}\right)-1\right)} \\
=\sum_{i=2, j=1}^{a_{i}, b_{j}} \sqrt{(b+3-1)(1-1)}+\sum_{i=2}^{a_{i}} \sqrt{(b+3-1)^{2}} \\
+\sum_{i=2}^{a_{i}} \sqrt{(a-2)(b+3-1)} \\
=a_{i}(b+2)+a_{i} \sqrt{(a-2)(b+2)} .
\end{gathered}
$$

Theorem 3.15: The Atom-bond connectivity index of a thorn wheel graph having $(a+$ $(a-1) b)$ number of vertices is

$$
A B C\left(W_{a, b}\right)=a_{i} b_{j} \sqrt{\frac{b+2}{(b+3)}}+a_{i} \frac{\sqrt{2(b+2)}}{(b+3)}+a_{i} \sqrt{\frac{a+b}{(a-1)(b+3)}}
$$

Proof: Here, we independently examine the pair of vertices of $W_{a}$ between the pair of vertices $u_{i}$ for $i=1,2,3, \ldots, b$ of $W_{a, b}$ along with the pair of vertices that belongs to $W_{a}$ and otherwise degree 1 vertex. Let $v_{1}, v_{2}, v_{3}, \ldots, v_{a}$ be the vertices of wheel graph $W_{a}$ where i ranges from $1,2,3,4, \ldots, a$ and let $u_{1}, u_{2}, u_{3}, \ldots, u_{b}$ be a degree one vertices of $W_{a, b}$ where $j=1,2,3, \ldots ., b$. (As in Figure. 6). Let $d\left(v_{i}\right)=(b+3)$ for $i=2,3,4, \ldots . a, d\left(v_{1}\right)=$ $(a-1)$ and $d\left(u_{j}\right)=1$. Then we have,

$$
\begin{aligned}
A B C\left(W_{a, b}\right) & =\sum_{i=2, j=1}^{a_{i}, b_{j}} \quad \sqrt{\frac{d\left(v_{i}\right)+d\left(u_{j}\right)-2}{d\left(v_{i}\right) d\left(u_{j}\right)}}+\sum_{i=2}^{a_{i}} \quad \sqrt{\frac{d\left(v_{i}\right)+d\left(v_{i}\right)-2}{d\left(v_{i}\right) d\left(v_{i}\right)}}+\sum_{i=2}^{a_{i}} \quad \sqrt{\frac{d\left(v_{1}\right)+d\left(v_{i}\right)-2}{d\left(v_{1}\right) d\left(v_{i}\right)}} \\
& =\sum_{i=2, j=1}^{a_{i}, b_{j}} \quad \sqrt{\frac{(b+3)+1-2}{(1)(b+3)}}+\sum_{i=2}^{a_{i}} \quad \sqrt{\frac{(b+3)+(b+3)-2}{(b+3)^{2}}}+\sum_{i=2}^{a_{i}} \quad \sqrt{\frac{(a-1)+(b+3)-2}{(a-1)(b+3)}} \\
& =a_{i} b_{j} \sqrt{\frac{b+2}{(b+3)}}+a_{i} \sqrt{\frac{2(b+2)}{(b+3)^{2}}}+a_{i} \sqrt{\frac{a+b}{(a-1)(b+3)}} \\
& =a_{i} b_{j} \sqrt{\frac{b+2}{(b+3)}}+a_{i} \frac{\sqrt{2(b+2)}}{(b+3)}+a_{i} \sqrt{\frac{a+b}{(a-1)(b+3)}}
\end{aligned}
$$

Theorem 3.16: The geometric-arithmetic index of a thorn wheel graph having $(a+$ $(a-1) b)$ number of vertices is 


$$
G A\left(W_{a, b}\right)=a_{i} b_{j} \frac{2 \sqrt{(b+3)}}{(b+4)}+a_{i}+a_{i} \frac{2 \sqrt{(a-1)(b+3)}}{(a+b+2)} .
$$

Proof: Here, we independently examine the pair of vertices of $W_{a}$ between the pair of vertices $u_{i}$, for $i=1,2,3, \ldots, b$ of $W_{a, b}$ along with the pair of vertices that belongs to $W_{a}$ and otherwise degree 1 vertex. Let $v_{1}, v_{2}, v_{3}, \ldots, v_{a}$ be the vertices of wheel graph $W_{a}$ where $i$ ranges from $1,2,3,4, \ldots, a$ and let $u_{1}, u_{2}, u_{3}, \ldots, u_{b}$ be a degree one vertices of $W_{a, b}$ where $j=1,2,3, \ldots, b$. (As in Figure. 6). Let $d\left(v_{i}\right)=(b+3)$ for $i=2,3,4, \ldots . a, d\left(v_{1}\right)=$ $(a-1)$ and $d\left(u_{j}\right)=1$. Then we have,

$$
\begin{aligned}
G A\left(W_{a, b}\right) & =\sum_{i=2, j=1}^{a_{i}, b_{j}} \quad \frac{2 \sqrt{d\left(v_{i}\right) d\left(u_{j}\right)}}{\left(d\left(v_{i}\right)+d\left(u_{j}\right)\right)}+\sum_{i=2}^{a_{i}} \quad \frac{2 \sqrt{d\left(v_{i}\right) d\left(v_{i}\right)}}{\left(d\left(v_{i}\right)+d\left(v_{i}\right)\right)}+\sum_{i=2}^{a_{i}} \quad \frac{2 \sqrt{d\left(v_{1}\right) d\left(v_{i}\right)}}{\left(d\left(v_{1}\right)+d\left(v_{i}\right)\right)} \\
& =\sum_{i=2, j=1}^{a_{i}, b_{j}} \frac{2 \sqrt{1(b+3)}}{(b+3+1)}+\sum_{i=2}^{a_{i}} \quad \frac{2 \sqrt{(b+3)(b+3)}}{((b+3)+(b+3))}+\sum_{i=2}^{a_{i}} \quad \frac{2 \sqrt{(a-1)(b+3)}}{((a-1)+(b+3))} \\
& =a_{i} b_{j} \frac{2 \sqrt{(b+3)}}{(b+4)}+a_{i} \frac{2 \sqrt{(b+3)^{2}}}{2(b+3)}+a_{i} \frac{2 \sqrt{(a-1)(b+3)}}{(a+b+2)} \\
& =a_{i} b_{j} \frac{2 \sqrt{(b+3)}}{(b+4)}+a_{i}+a_{i} \frac{2 \sqrt{(a-1)(b+3)}}{(a+b+2)}
\end{aligned}
$$

3.1.8. A Cog-Wheel graph $W^{c}{ }_{a}$.

$W_{a}^{c}$ is the graph formed from a star graph $W_{a}(a \geq 4)$ of the vertex set $\left\{v_{1}, v_{2}, v_{3}, \ldots, v_{a-1}, v_{a}\right\}$ with the addition of $(b-1)$ number of vertices such as $\left\{u_{1}, u_{2}, u_{3}, \ldots, u_{b-1}\right\}$ and edges given by $\left\{u_{j} v_{i+1}, u_{j} v_{i+2}: i=1,2,3, \ldots\right.$, a and $j=$ $1,2,3, \ldots \ldots,(b-1)\}\left(v_{a+1}=v_{2}\right)$, as shown in Figure 7 .

We know that $p\left(W^{c} a\right)=(a+b-1), q\left(W_{a}^{c}\right)=2(a+b-2)$.

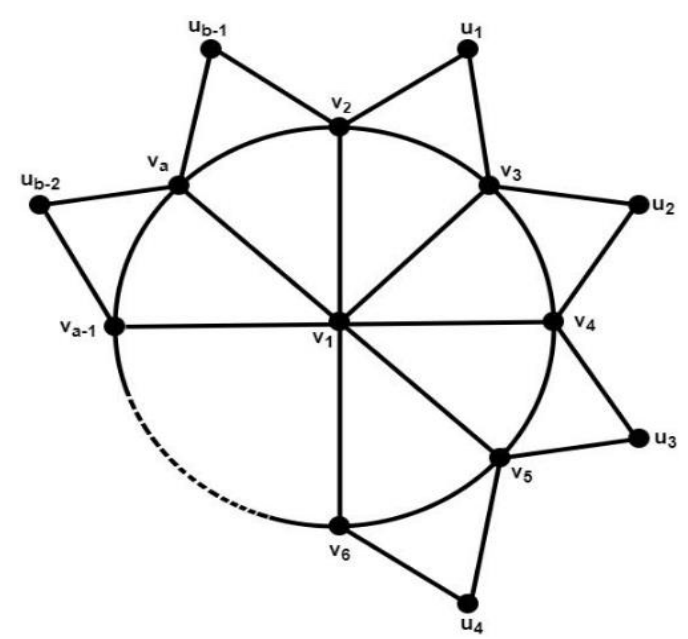

Figure 7. A Cog-Wheel graph $W_{a}^{c}$.

3.1.9. The thorn cog-wheel graph $W^{c^{*}} a$.

$W^{c^{*}} a$ is the graph formed from a $W_{a}^{c}(a \geq 4)$, of the vertex set $\left\{v_{1}, v_{2}, v_{3}, \ldots, v_{a-1}, v_{a}, u_{1}, u_{2}, u_{3}, \ldots ., u_{b-1}\right\}$ for $i=1,2,3, \ldots$, a and $j=1,2,3, \ldots \ldots,(b-$ 
1) with the addition of $2(b-1)$ number of vertices such as $\left\{w_{1}, w_{2}, w_{3}, \ldots ., w_{2 b-3}, w_{2 b-2}\right\}$ and edges given by $\left\{u_{j} w_{2 j-1}, u_{j} w_{2 j}: j=1,2,3, \ldots \ldots,(b-1)\right\}$, as shown in Figure 8 .

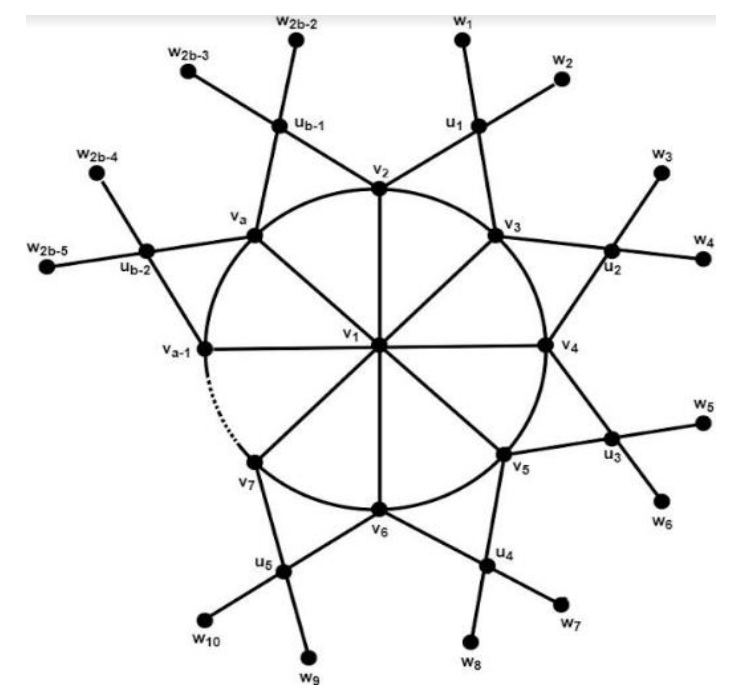

Figure 8. The Thorn Cog-Wheel graph $W^{c^{*}}{ }_{a}$.

Theorem 3.17: The reciprocal randic index of a thorn cog-wheel graph having $(a+$ $3(b-1))$ number of vertices is

$$
R^{-1}\left(W^{c^{*}} a\right)=2 a_{i}\left(b_{j}-1\right) \sqrt{5}+a_{i} \sqrt{5(a-1)}+a_{i} 5+4\left(b_{j}-1\right)\left(b_{k}-1\right) .
$$

Proof: Here we independently examine the pair of vertices of $W_{a}$ between the pair of vertices $u_{j}$ for $j=1,2,3, \ldots,(b-1)$ of $W^{c}{ }_{a}$ along with the pair of vertices that belongs to $W_{a}$ and between the pair of vertices $u_{j}$ for $j=1,2,3, \ldots,(b-1)$ of $W^{c}{ }_{a}$ along with the pair of vertices degree 1 vertices of $W^{c^{*}}{ }_{a}$. Let $v_{1}, v_{2}, v_{3}, \ldots, v_{a}$ be the vertices of wheel graph $W_{a}$, where $i$ ranges from $1,2,3,4, \ldots, a$ and let $u_{1}, u_{2}, u_{3}, \ldots, u_{b-1}$ be addition of $(b-1)$ number of vertices of $W_{a}^{c}$ where $j=1,2,3, \ldots,(b-1)$. Also, let $w_{1}, w_{2}, \ldots \ldots, w_{2 b-2}, k=$ $1,2,3, \ldots,(2 b-2)$ of $W^{c^{*}} a$ be an additional $(2 b-2)$ number of degree 1 vertices of $W^{c^{*}}{ }_{a}$. (As in Figure. 8). Let $d\left(v_{1}\right)=(a-1), d\left(u_{j}\right)=4($ for $j=1,2, \ldots,(b-1)), d\left(v_{i}\right)=$ $5($ for $i=2,3,4, \ldots \ldots, a)$ and $d\left(w_{k}\right)=1($ for $k=1,2,3, \ldots \ldots, 2(b-1))$. Then we have,

$$
\begin{aligned}
& R^{-1}\left(W^{c^{*}}{ }_{a}\right)=\sum_{i=2, j=1}^{a_{i,}\left(b_{j}-1\right)} \quad \sqrt{\left(d\left(v_{i}\right)\right)\left(d\left(u_{j}\right)\right)}+ \\
& \sum_{i=2}^{a_{i}} \sqrt{\left(d\left(v_{1}\right)\right)\left(d\left(v_{i}\right)\right)}+\sum_{i=2}^{a_{i}} \sqrt{\left(d\left(v_{i}\right)\right)^{2}}+ \\
& \sum_{j, k=1}^{b_{j}-1,2 b_{k}-2} \sqrt{\left(d\left(u_{j}\right)\right)\left(d\left(w_{k}\right)\right)} \\
& =\sum_{i=2, j=1}^{a_{i},\left(b_{j}-1\right)} \quad \sqrt{5(4)}+\sum_{i=2}^{a_{i}} \quad \sqrt{5(a-1)}+\sum_{i=2}^{a_{i}} \quad \sqrt{(5)^{2}}+ \\
& \sum_{j, k=1}^{b_{j}-1,2 b_{k}-2} \sqrt{4(1)} \\
& =a_{i}\left(b_{j}-1\right) 2 \sqrt{5}+a_{i} \sqrt{(a-1) 5}+a_{i} 5+\left(b_{j}-1\right)\left(2 b_{k}-2\right) 2 \\
& =2 a_{i}\left(b_{j}-1\right) \sqrt{5}+a_{i} \sqrt{5(a-1)}+a_{i} 5+4\left(b_{j}-1\right)\left(b_{k}-1\right) .
\end{aligned}
$$


Theorem 3.18: The reduced reciprocal randic index of a thorn cog-wheel graph having $(a+3(b-1))$ number of vertices is

$$
R R^{-1}\left(W^{c^{*}} a\right)=2 a_{i}\left(b_{j}-1\right) \sqrt{3}+2 a_{i} \sqrt{(a-2)}+4 a_{i}
$$

Proof: Here we independently examine the pair of vertices of $W_{a}$ between the pair of vertices $u_{j}$ for $j=1,2,3, \ldots,(b-1)$ of $W^{c}{ }_{a}$ along with the pair of vertices that belongs to $W_{a}$ and between the pair of vertices $u_{j}$ for $j=1,2,3, \ldots,(b-1)$ of $W^{c}{ }_{a}$ along with the pair of vertices degree 1 vertices of $W^{c^{*}}{ }_{a}$. Let $v_{1}, v_{2}, v_{3}, \ldots, v_{a}$ be the vertices of wheel graph $W_{a}$ where $i$ ranges from $1,2,3,4, \ldots, a$ and let $u_{1}, u_{2}, u_{3}, \ldots, u_{b-1}$ be addition of $(b-1)$ number of vertices of $W^{c}{ }_{a}$ where $j=1,2,3, \ldots,(b-1)$. Also, let $w_{1}, w_{2}, \ldots \ldots, w_{2 b-2}, k=$ $1,2,3, \ldots .,(2 b-2)$ of $W^{c^{*}}$ be an additional $(2 b-2)$ number of degree 1 vertices of $W^{c^{*}} a$. (As in Figure. 8). Let $d\left(v_{1}\right)=(a-1), d\left(u_{j}\right)=4($ for $j=1,2, \ldots,(b-1)), d\left(v_{i}\right)=$ $5($ for $i=2,3,4, \ldots, a)$ and $d\left(w_{k}\right)=1($ for $k=1,2,3, \ldots \ldots, 2(b-1))$. Then we have,

$$
\begin{gathered}
R R^{-1}\left(W^{c^{*}}{ }_{a}\right)=\sum_{i=2, j=1}^{a_{i},\left(b_{j}-1\right)} \sqrt{\left(d\left(v_{i}\right)-1\right)\left(d\left(u_{j}\right)-1\right)}+\sum_{i=2}^{a_{i}} \sqrt{\left(d\left(v_{1}\right)-1\right)\left(d\left(v_{i}\right)-1\right)} \\
\quad+\sum_{i=2}^{a_{i}} \sqrt{\left(d\left(v_{i}\right)-1\right)^{2}}+ \\
\sum_{j, k=1}^{b_{j}-1,2 b_{k}-2} \sqrt{\left(d\left(u_{j}\right)-1\right)\left(d\left(w_{k}\right)-1\right)}
\end{gathered}
$$

$$
\begin{aligned}
& =\sum_{i=2, j=1}^{a_{i},\left(b_{j}-1\right)} \sqrt{(5-1)(4-1)}+\sum_{i=2}^{a_{i}} \sqrt{(a-2) 4} \\
& \quad+\sum_{i=2}^{a_{i}} \sqrt{(4)^{2}}+\sum_{j, k=1}^{b_{j}-1,2 b_{k}-2} \sqrt{3(0)} \\
& =2 a_{i}\left(b_{j}-1\right) \sqrt{3}+2 a_{i} \sqrt{(a-2)}+4 a_{i} .
\end{aligned}
$$

Theorem 3.19: The Atom-bond connectivity index of a thorn cog-wheel graph having $(a+3(b-1))$ number of vertices is

$$
A B C\left(W^{c^{*}} a\right)=a_{i}\left(b_{j}-1\right) \frac{\sqrt{7}}{2 \sqrt{5}}+a_{i} \sqrt{\frac{a+2}{5(a-1)}}+a_{i} \frac{2 \sqrt{2}}{5}+\left(b_{j}-1\right)\left(b_{k}-1\right) \sqrt{3} .
$$

Proof: Here we independently examine the pair of vertices of $W_{a}$ between the pair of vertices $u_{j}$ for $j=1,2,3, \ldots,(b-1)$ of $W^{c}{ }_{a}$ along with the pair of vertices that belongs to $W_{a}$ and between the pair of vertices $u_{j}$ for $j=1,2,3, \ldots,(b-1)$ of $W^{c}{ }_{a}$ along with the pair of vertices degree 1 vertices of $W^{c^{*}}{ }_{a}$. Let $v_{1}, v_{2}, v_{3}, \ldots, v_{a}$ be the vertices of wheel graph $W_{a}$ where $i$ ranges from $1,2,3,4, \ldots, a$ and let $u_{1}, u_{2}, u_{3}, \ldots, u_{b-1}$ be addition of $(b-1)$ number of vertices of $W^{c}{ }_{a}$ where $j=1,2,3, \ldots,(b-1)$. Also, let $w_{1}, w_{2}, \ldots \ldots, w_{2 b-2}, k=$ $1,2,3, \ldots .,(2 b-2)$ of $W^{c^{*}} a$ be an additional $(2 b-2)$ number of degree 1 vertices of $W^{c^{*}} a$. (As in Figure. 8). Let $d\left(v_{1}\right)=(a-1), d\left(u_{j}\right)=4($ for $j=1,2, \ldots,(b-1)), d\left(v_{i}\right)=$ $5($ for $i=2,3,4, \ldots . ., a)$ and $d\left(w_{k}\right)=1($ for $k=1,2,3, \ldots \ldots, 2(b-1))$. Then we have, 


$$
\begin{aligned}
& A B C\left(W^{c^{*}}\right)=\sum_{i=2, j=1}^{a_{i}\left(b_{j}-1\right)} \quad \sqrt{\frac{d\left(v_{i}\right)+d\left(u_{j}\right)-2}{d\left(v_{i}\right) d\left(u_{j}\right)}}+ \\
& \sum_{i=2}^{a_{i}} \quad \sqrt{\frac{d\left(v_{1}\right)+d\left(v_{i}\right)-2}{d\left(v_{1}\right) d\left(v_{i}\right)}}+\sum_{i=2}^{a_{i}} \quad \sqrt{\frac{d\left(v_{i}\right)+d\left(v_{i}\right)-2}{d\left(v_{i}\right) d\left(v_{i}\right)}}+ \\
& \sum_{j, k=1}^{b_{j}-1,2 b_{k}-2} \sqrt{\frac{d\left(u_{j}\right)+d\left(w_{k}\right)-2}{d\left(u_{j}\right) d\left(w_{k}\right)}} \\
& =\sum_{i=2, j=1}^{a_{i},\left(b_{j}-1\right)} \quad \sqrt{\frac{5+4-2}{5(4)}}+\sum_{i=2}^{a_{i}} \quad \sqrt{\frac{(a-1)+(5)-2}{5(a-1)}}+ \\
& \sum_{i=2}^{a_{i}} \quad \sqrt{\frac{(5)+(5)-2}{5(5)}}+\sum_{j, k=1}^{b_{j}-1,2 b_{k}-2} \quad \sqrt{\frac{4+1-2}{4(1)}} \\
& =a_{i}\left(b_{j}-1\right) \sqrt{\frac{7}{4(5)}}+a_{i} \sqrt{\frac{a+2}{5(a-1)}}+a_{i} \frac{2 \sqrt{2}}{5}+\left(b_{j}-1\right)\left(2 b_{k}-2\right) \frac{\sqrt{3}}{2} \\
& =a_{i}\left(b_{j}-1\right) \frac{\sqrt{7}}{2 \sqrt{5}}+a_{i} \sqrt{\frac{a+2}{5(a-1)}}+a_{i} \frac{2 \sqrt{2}}{5}+\left(b_{j}-1\right)\left(b_{k}-1\right) \sqrt{3} .
\end{aligned}
$$

Theorem 3.20: The geometric-arithmetic index of a thorn cog-wheel graph having $(a+3(b-1))$ number of vertices is

$$
G A\left(W^{c^{*}}{ }_{a}\right)=a_{i}\left(b_{j}-1\right) \frac{4 \sqrt{5}}{9}+a_{i} \frac{2 \sqrt{5(a-1)}}{(a+4)}+a_{i}+\frac{8\left(b_{j}-1\right)\left(b_{k}-1\right)}{5} .
$$

Proof: Here, we independently examine the pair of vertices of $W_{a}$ between the pair of vertices $u_{j}$ for $j=1,2,3, \ldots,(b-1)$ of $W^{c}{ }_{a}$ along with the pair of vertices that belongs to $W_{a}$ and between the pair of vertices $u_{j}$ for $j=1,2,3, \ldots,(b-1)$ of $W^{c}{ }_{a}$ along with the pair of vertices degree 1 vertices of $W^{c^{*}}{ }_{a}$. Let $v_{1}, v_{2}, v_{3}, \ldots, v_{a}$ be the vertices of wheel graph $W_{a}$ where $i$ ranges from $1,2,3,4, \ldots, a$ and let $u_{1}, u_{2}, u_{3}, \ldots, u_{b-1}$ be addition of $(b-1)$ number of vertices of $W_{a}^{c}$ where $j=1,2,3, \ldots,(b-1)$. Also, let $w_{1}, w_{2}, \ldots \ldots, w_{2 b-2}, k=$ $1,2,3, \ldots,(2 b-2)$ of $W^{c^{*}}$ a be an additional $(2 b-2)$ number of degree 1 vertices of $W^{c^{*}}{ }_{a}$. (As in Figure. 8). Let $d\left(v_{1}\right)=(a-1), d\left(u_{j}\right)=4($ for $j=1,2, \ldots,(b-1)), d\left(v_{i}\right)=$ $5($ for $i=2,3,4, \ldots . ., a)$ and $d\left(w_{k}\right)=1($ for $k=1,2,3, \ldots \ldots, 2(b-1))$. Then we have,

$$
\begin{aligned}
G A\left(W^{c^{*}}{ }_{a}\right)= & \sum_{i=2, j=1}^{a_{i},\left(b_{j}-1\right)} \quad \frac{2 \sqrt{d\left(v_{i}\right) d\left(u_{j}\right)}}{d\left(v_{i}\right)+d\left(u_{j}\right)}+\sum_{i=2}^{a_{i}} \quad \frac{2 \sqrt{d\left(v_{1}\right) d\left(v_{i}\right)}}{d\left(v_{1}\right)+d\left(v_{i}\right)}+\sum_{i=2}^{a_{i}} \quad \frac{2 \sqrt{\left(d\left(v_{i}\right)\right)^{2}}}{d\left(v_{i}\right)+d\left(v_{i}\right)} \\
& +\sum_{j, k=1}^{b_{j}-1,2 b_{k}-2} \quad \frac{2 \sqrt{d\left(u_{j}\right) d\left(w_{k}\right)}}{d\left(u_{j}\right)+d\left(w_{k}\right)} \\
= & \sum_{i=2, j=1}^{a_{i},\left(b_{j}-1\right)} \frac{2 \sqrt{5(4)}}{(5+4)}+\sum_{i=2}^{a_{i}} \quad \frac{2 \sqrt{5(a-1)}}{(a-1+5)}+\sum_{i=2}^{a_{i}} \quad \frac{2 \sqrt{(5)^{2}}}{10} \\
& +\sum_{j, k=1}^{b_{j}-1,2 b_{k}-2} \\
= & a_{i}\left(b_{j}-1\right) \frac{4 \sqrt{5}}{9}+a_{i} \frac{2 \sqrt{5(a-1)}}{(a+4)}+a_{i}+\left(b_{j}-1\right)\left(2 b_{k}-2\right) \frac{4}{5}
\end{aligned}
$$




$$
=a_{i}\left(b_{j}-1\right) \frac{4 \sqrt{5}}{9}+a_{i} \frac{2 \sqrt{5(a-1)}}{(a+4)}+a_{i}+\frac{8\left(b_{j}-1\right)\left(b_{k}-1\right)}{5} .
$$

\section{Conclusions}

In this paper, we have computed reciprocal randic $\left(R^{-1}\right)$ index, reduced reciprocal $\operatorname{randic}\left(R R^{-1}\right)$ index, atom-bond connectivity $(A B C)$ index, geometric-arithmetic $(G A)$ index of complete thorn graph and wheel graph along with its special cases. These descriptors constitute the biological and physicochemical properties of chemical compounds. Topological indices have many applications in the field of modern chemistry.

\section{Funding}

This research received no external funding.

\section{Acknowledgments}

We are grateful to our institutes for their support.

\section{Conflicts of Interest}

The authors declare no conflict of interest.

\section{References}

1. Gutman, I. Distance in thorny graph. Publ. Inst. Math. Beograd 1998, 63, 31-36.

2. Knor, M.; Potcnik, P.; Skrekovski, R. Relationship between the edge-Winer index and the Gutman index of a graph. Discrete Appl. Math. 2014, 167, 197-201, https://doi.org/10.1016/j.dam.2013.12.009.

3. Vukicevic, D.; Graovac, A. On modified Wiener indices of thorn graphs. MATCH - Communications in Mathematical and in Computer Chemistry 2004, 50, 93-108.

4. Vukicevic, D.; Nikolić, S.; Trinajstić, N. On the Schultz Index of Thorn Graphs. Internet Electronic Journal of Molecular Design 2005, 4, 501-514.

5. Zhou, B. On modified Wiener indices of thorn trees. Kragujevac J. Math. 2005, 27, 5-9.

6. Wu, B. Wiener index of line graphs. MATCH Commun. Math. Comput. Chem. 2010, 64, 699-706.

7. Wei, J.; Imran, M.; Iqbal, M.A.; Zaighum, M.A. Zagreb-Type Indices of-Vertex Join and-Edge Join of Graphs. Journal of Chemistry 2020, 2020, https://doi.org/10.1155/2020/9767128.

8. Li, Q.Z.; Nazar, K.; Ahmed, I.; Tlili, I. Valency-Based Descriptors for Silicon Carbides, Bismuth (III) Iodide, and Dendrimers in Drug Applications. Journal of Chemistry 2020, 2020, https://doi.org/10.1155/2020/8616309.

9. Pio, J.; Thayamathy, Panchadcharam, E.; Masilamani, K. Computing Zagreb and Randic Indices of PEGCored Dendrimers Used for Drug and Gene Delivery. Journal of Chemistry 2020, 2020, https://doi.org/10.1155/2020/2821510.

10. Huang, Y.; Mutee-ur-Rehman, H.; Nazeer, S.; Afzal, D.; Qiang, X. Some Bounds of Weighted Entropies with Augmented Zagreb Index Edge Weights. Discrete Dynamics in Nature and Society 2020, 2020, https://doi.org/10.1155/2020/3562382.

11. Kwun, Y.C.; Rehman, H.M.u.; Yousaf, M.; Nazeer, W.; Kang, S.M. The Entropy of Weighted Graphs with Atomic Bond Connectivity Edge Weights. Discrete Dynamics in Nature and Society 2018, 2018, https://doi.org/10.1155/2018/8407032.

12. Li, X.; Ahmad, M.; Javaid, M.; Saeed, M.; Liu, J.-B. Bounds on General Randic Index for F-Sum Graphs. Journal of Mathematics 2020, 2020, https://doi.org/10.1155/2020/9129365.

13. Imran, M.; Akhter, S.; Iqbal, Z. On the Eccentric Connectivity Polynomial of $\mathscr{F}$-Sum of Connected Graphs. Complexity 2020, 2020, https://doi.org/10.1155/2020/5061682.

14. Tang, J.-H.; Ali, U.; Javaid, M.; Shabbir, K. Zagreb Connection Indices of Subdivision and Semi-Total Point Operations on Graphs. Journal of Chemistry 2019, 2019, https://doi.org/10.1155/2019/9846913. 